\title{
Control and Anticontrol of Chaos for Arrhythmia Based on Impulsive Differential Theory
}

\author{
Lihua Miao \\ Department of Mathematics \& Physics, Shenyang Medical College \\ miaolihua@163.com
}

\begin{abstract}
The chaotic dynamic characteristic of arrhythmia is analyzed and the control and anticontrol of chaos in arrhythmia are presented in this paper. First, the cardiac chaotic dynamics can be established based on the clinical data between heart RR signals using statistical modeling approach. Then, this paper utilizes the advantages of approximation accuracy of artificial neural network to simulate cardiac output between the RR signals. Finally, based on the impulsive differential theory, we put forward a novel chaotic control and anticontrol algorithm to deal with cardiac dynamics model between RR output signals to eliminate cardiac arrhythmias.
\end{abstract}

Keywords: Chaotic control, Anti-control of chaos, Arrhythmia, BP neural networks, Impulsive differentiation

\section{Introduction}

As is well-known, heart is one of the most complicated dynamic systems. The ECG signal between the RR is one of the important information which reflects the various conditions of cardiac activity. Recent studies show that healthy heart dynamics may be in the edge of chaos. The relevant researches have shown that the chaotic behavior of the healthy heart weakens with increasing age [1]. Moreover, the chaos of atrial fibrillation patients is the weakest. It suggested that the heart variability reduction and periodic enhancement is often associated with diseases.

Chaos is a deterministic dynamics system in a seemingly random movement, whose essence is the extreme sensitivity of long-term behavior to initial conditions. There are many chaotic phenomena in real systems, such as the human brain and heart activity [2-6]. Chaos theory belongs to the nonlinear science. Only nonlinear system can produce chaotic motion. In recent thirty years, with the maturing of chaos theory, people are no longer satisfied with just understand the chaotic phenomenon. More chaotic systems have been controlled in order to make use of the chaos better, so as a new research direction of chaos control arises at the historic moment and become a research hotspot in the field of nonlinear science. Here, the chaotic control is a broad concept, including chaotic control, chaotic synchronization and the third aspects of this content, so-called chaotic anticontrol. i.e., the meaning of anticontrol is to make a non-chaotic system chaotic or enhance an existing chaotic behavior of chaotic systems. Chaotic control research started relatively late. Moreover, chaotic anticontrol problem is put forward in some cases, where the chaos phenomenon is useful or necessary. As to cardiac dynamics research, people found the normal state of the dynamic behavior of the heart has a certain form of chaos. The pathological chaotic disappearing is early signs of heart disease. In such circumstances, it is necessary to maintain or enhance the chaotic behavior, in order to maintain the normal function of the heart. Especially it is worthy noting that some biological systems need to have the complexity of chaos in order to work properly. The chaos disappears 
pathologically may mean a heart attack or stroke. Therefore, it is necessary to study the method systematically how to produce or maintain chaotic behavior.

In 1992, people began to develop the arrhythmia chaotic control problem research. Alan etc. of California University studied the isolation region on rabbit heart. Ouabainadrenal hormone was injected into the coronary artery, and then, it causes the rapid myocardial irregular shrinkage. After the appearance of symptom of the arrhythmia, they used the proportional perturbation feedback (PPF) control chaos method to suppress arrhythmia. The experimental results show that these random control signals seemly make heartbeat regular enough and can bring the heart rate down to normal level. However, the random signal or periodic signal sometimes cannot terminate the arrhythmia, and often makes the condition worse. Then they had to try to employ different forms of OGY control scheme to control human arrhythmia. It is expected that this control technology can be used for the treatment of cardiac fibrillation. But there is little literature in this area so far. Substantive breakthroughs in research have not been reported. For this, based on the impulsive differential theory, chaotic control and anticontrol methods for arrhythmia are put forward in this paper. It provides important theoretical basis for the development of medical equipment, such as automatic cardioerter defibrillator to treat arrhythmia disease. That is, we propose a chaotic anticontrol method based on the impulsive differential theory. The original dynamics exhibit strong periodicity and chaos is very weak, the proposed anticontrol method can making it similar to a healthy heart. This is a very frontier subject and has very important theoretical research value.

\section{Chaotic Control and Anticontrol Strategy for Arrhythmia}

The main content of this paper contains arrhythmia chaotic control and anticontrol approach study. The choices between the ECG RR signals are determined as the startpoint of the research. A variety of nonlinear signal processing theory of RR interval signal analysis is employed to prove the existence of a decisive chaotic phenomenon. Then, the RR interval signals are taken as samples to train the neural networks, which have an analog signal between RR cardiac output function. Finally, we propose a novel chaotic control and anticontrol method based on impulsive differential theory in order to treat the arrhythmia.

Especially, chaotic anticontrol strategy is given below. First, the simplest onedimensional continuous linear system (often expressed by one-dimensional linear differential equations) is started by adding a nonlinear feedback delay term. The controlled system becomes infinite dimensional, and then the necessary conditions to produce chaotic behavior is satisfied. The Pan functional differential equation theory is utilized to carry out theoretical analysis of the stability of dynamic behavior of the controlled system. The chaotic identification and prediction algorithm to determine the conditions of chaotic occurrence is put forward to analyze the impact of control parameters and time delay of bifurcation and chaos. As to multidimensional continuous dynamic systems, some representative examples are adopted by non-time-delay feedback and anti-feedback control implementation from stable and unstable equilibrium point, limit cycle to multi-cycle solution. As to discrete dynamic system, it can draw on the idea of chaos control method for continuous system (such as perturbation parameters, pulse control, etc.,) to achieve anticontrol result.

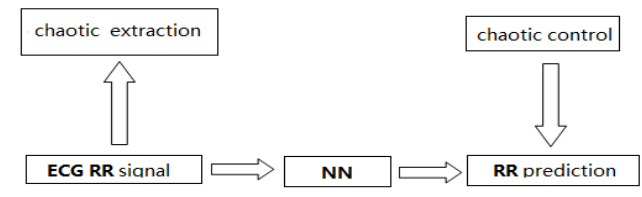

Figure 1. The Schematic Diagram of Chaotic Control and Anticontrol Strategy for Arrhythmia 


\section{Back Propagation Neural Network Modeling Process}

The error back propagation neural network (BP for short), which is used to simulate the cardiac kinetics characteristics, and the network is multi-layer forward network of a one-way transmission. It has successfully solved the multilayer network learning problems of connection weights for the hidden layer neurons. Figure 2 is structural diagram of BP neural network. The first layer of the figure is the input layer and there are $M$ input nodes. The output of input layer nodes is equal to the input, and the last layer is the output layer, who has $p$ output nodes. The intermediate layer is the hidden layer and there are $n$ nodes. $u_{i j}$ is the connection weights value between the input layer and hidden layer nodes. $u_{j k}$ is the connection weights value between the hidden layer and output layer nodes. The input of the hidden layer and output layer nodes are the weighted value of the output of the previous layer nodes. The incentive degree of each node is decided by its excitation function [7-9].

The basic idea of error back propagation learning algorithm is the least square algorithm. It calculates derivative of error of each weight change and adjustment of each unit weights to reduce the error between actual output value and the desired output value.

The learning process of BP algorithm is composed of forward and backward propagation. In the forward propagation, the information from the input layer is handled through the hidden layer step by step and transmitted to output layer. The neurons in each layer are only affected by their former neurons' state. If the output of output layer and the expected output do not match, then the algorithm goes to the back propagation procedure. The error signal returns along the same route and minimizes the error signal by modifying the weights of each layer of neurons.

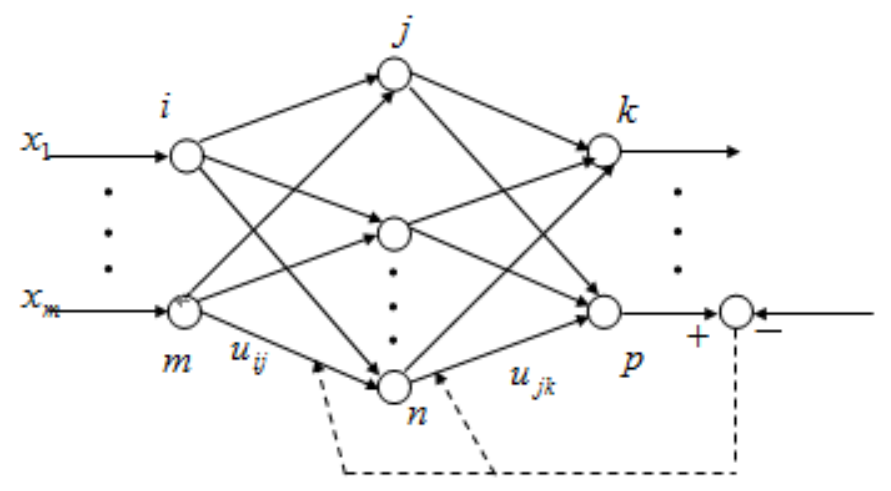

Figure 2. The Layer Structure of BP Neural Networks

(1) Calculation steps of BP learning algorithm

In the feedforward network layer shown in Figure 2, the total weighted of input layer and output layer of any $k$ node is:

$$
x_{k}=\sum_{j=1}^{n} u_{j k} y_{j}
$$

where $k, j$ are nodes of output layer and the front layer respectively, $n$ is the number of former layer nodes, $u_{j k}$ is the connection weights of former layer of the first $j$ th node and the $k$ th node in the output layer, $y_{j}$ is the output of the first $j$ th node in the former layer. The transfer function of neurons is chosen as Sigmoid function, then the output of the first $k$ node is:

$$
y_{k}=\frac{1}{1+e^{-\beta x_{k}}}
$$


After determining the outputs of all the output units, the definition of network error calculation formula is:

$$
E=\frac{1}{2} \sum_{k=1}^{P}\left(y_{k}-d_{k}\right)^{2}
$$

where $P$ is the number of output layer nodes, $y_{k}$ is output of the output layer of the first $k$ node, $d_{k}$ is the expected output of the first $k$ node. The steps of back propagation algorithm calculation are presented as follows:

(a) Calculate the difference between actual output of the output nodes and the expected output. That is the derivative of the error changes of the node output.

$$
\frac{\partial E}{\partial y_{k}}=y_{k}-d_{k}
$$

(b) Calculating total weighted input change of an output node, the error derivative is

$$
\frac{\partial E}{\partial x_{k}}=\frac{\partial E}{\partial y_{k}} \cdot \frac{\partial y_{k}}{\partial x_{k}}=\left(y_{k}-d_{k}\right) y_{k}\left(1-y_{k}\right)
$$

where

$$
\frac{\partial y_{k}}{\partial x_{k}}=y_{k}\left(1-y_{k}\right)
$$

(c) Calculate error change rate of an output node connection weights

$$
\frac{\partial E}{\partial u_{j k}}=\frac{\partial E}{\partial x_{k}} \cdot \frac{\partial x_{k}}{\partial u_{j k}}=\left(y_{k}-d_{k}\right) y_{k}\left(1-y_{k}\right) y_{j}
$$

where

$$
\frac{\partial x_{k}}{\partial u_{j k}}=y_{j}
$$

(d) Calculate error change rate of each node that changes output layer connected with former layer, and set its value stack.

$$
\sum_{j=1}^{n} \frac{\partial E}{\partial y_{j}}=\sum_{j=1}^{n} \frac{\partial E}{\partial x_{k}} \cdot \frac{\partial x_{k}}{\partial y_{j}}=\sum_{j=1}^{n}\left(y_{k}-d_{k}\right) y_{k}\left(1-y_{k}\right) u_{j_{k}}
$$

Repeat the steps (b)-(d) until the minimum error.

(2) The computer process of learning algorithm of BP network

(a) Initialization. For all the weights assigned to a smaller random number, and set the initial value of the threshold.

(b) The training data set is provided. The input and the desired output vector are given as $X=\left(x_{1}, x_{2}, \ldots, x_{\mathrm{m}}\right)$ and $D=\left(d_{1}, d_{2}, \ldots, d_{\mathrm{p}}\right)$.

(c) Calculate the actual output.

$$
y_{k}=f\left(\sum u_{j k} x_{j}\right)
$$

where $f$ is the $S$ function.

$$
f(x)=\frac{1}{1+e^{-(x-\theta)}}
$$


(d) Revise weighted coefficient. The error signal from the output terminal sets back propagation to the hidden layer, and the weights are corrected according to the following formula in the process of communication.

$$
u_{j k}(t+1)=u_{j k}(t)+\lambda b_{k} y_{j}
$$

where $\lambda>0$ is the gain, $b_{k}$ the error of the $k$ th node.

For the $k$ th output node, the error is

$$
b_{k}=y_{k}\left(1-y_{k}\right)\left(d_{k}-y_{k}\right)
$$

When using the pulse, the adjusting weight formula turns into the following formula

$$
u_{j k}(t+1)=u_{j k}(t)+\lambda b_{j} y_{j}+\alpha\left[u_{j k}(t)-u_{j k}(t-1)\right]
$$

where $\alpha$ is the momentum factor, $0<\alpha<1$.

(e) Go back to (b) and repeat the procedure until the error requirement is satisfied.

\section{Impulsive Differential Control Method}

After the neural network model of cardiac nonlinear dynamic system was constructed, we consider the method of impulse control as follow.

$$
\begin{array}{ll}
\dot{x}=A f(x)+B u, & t \neq \tau_{k} \\
\Delta x=I_{k}(x(t)), & t=\tau_{k} \\
x\left(t_{0}+\right)=x_{0}, & t_{0} \geq 0, k=1,2,3 \cdots
\end{array}
$$

where $I_{k}(x(t))(k=1,2,3 \cdots)$ is the smooth vector function defined on $D \subset R^{n}$, $t_{0}=\tau_{0}<\tau_{1}<\cdots<\tau_{k}, \tau_{k}-\tau_{k-1}=T, \lim _{k \rightarrow+\infty} \tau_{k}=+\infty, \Delta x=x\left(\tau_{k}+\right)-x\left(\tau_{k}-\right), x\left(\tau_{k}-\right)$ and $x\left(\tau_{k}+\right)$ are left and right limit respectively at $t=\tau_{k}$. If $(A, B)$ is controlled, the feedback control can be designed according to the following formula:

$$
I_{k}(x(t))=y_{k}-x(t-), \quad t=\tau_{k}, \quad k=1,2,3 \cdots
$$

\section{Anticontrol of Chaos for Arrhythmia}

For the problem of chaotic anticontrol, we use the Poincare map, which can put the $n$ dimensional continuous nonlinear dynamic model transform into the $n-1$ dimensional discrete system, and therefore the question becomes simple. The Poincare map is an important tool in the research of chaos.

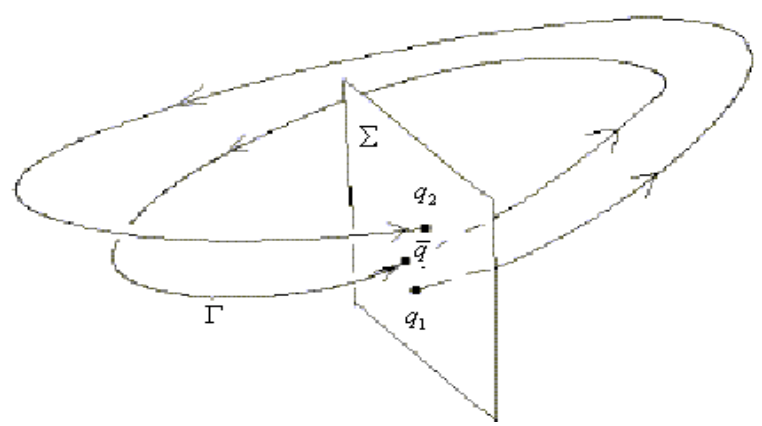

Figure 3 Poincare Map 
Then, we consider abovementioned $n$-dimensional discrete-time autonomous system with the form

$$
x(k+1)=g(x(k))
$$

where $g$ is a $C^{1}$ nonlinear map. Let $g^{t}$ denote the $t$ times of compositions of $g$ with itself. A point, $x^{*}$, is said to be a $p$-periodic point of $g$ if $g^{p}\left(x^{*}\right)=x^{*}$ but $g^{t}\left(x^{*}\right) \neq x^{*}$ for $p \geq t \geq 1$. If $p=1$, i.e., $g\left(x^{*}\right)=x^{*}, x^{*}$ is called a fixed point. Let $g^{\prime}(x)$ and $\operatorname{det}\left(g^{\prime}(x)\right)$ be the Jacobian of $g$ at the point $x$ and its determinant respectively.

Definition 1 [10] Suppose $\boldsymbol{x}^{*}$ is a fixed point of $\boldsymbol{g}$ with all eigenvalues of $\boldsymbol{g}^{\prime}\left(\boldsymbol{x}^{*}\right)$ exceeding 1 in magnitude, and suppose there exists a point $x^{0} \neq x^{*}$ in a repelling neighborhood of $\boldsymbol{x}^{*}$, such that for some positive integer $m, \boldsymbol{g}^{m}\left(\boldsymbol{x}^{0}\right)=\boldsymbol{x}^{*}$ and $\operatorname{det}\left(\left(\boldsymbol{g}^{m}\right)^{\prime}\left(\boldsymbol{x}^{0}\right)\right) \neq 0$. Then $\boldsymbol{x}^{*}$ is said to be a snap-back repeller of $\boldsymbol{g}$.

Lemma 1 If system (3) has a snap-back repeller then the system is chaotic in the sense of Li-Yorke, namely,

(1) there exists a positive integer $n$ such that for every integer $p \geq n$, system (3) has $p$ periodic points;

(2)there exists a scrambled set (an uncountable invariant set $S$ containing no periodic points) such that

(a) $\boldsymbol{g}(S) \subset S$,

(b) for every $\boldsymbol{y} \in S$ and any periodic point $\boldsymbol{x}$ of (3)

$$
\lim _{k \rightarrow \infty} \sup \left\|\boldsymbol{g}^{k}(\boldsymbol{x})-\boldsymbol{g}^{k}(\boldsymbol{y})\right\|>0,
$$

(c) for every $\boldsymbol{x}, \boldsymbol{y} \in S$ with $\boldsymbol{x} \neq \boldsymbol{y}$

$$
\lim _{k \rightarrow \infty} \sup \left\|\boldsymbol{g}^{k}(\boldsymbol{x})-\boldsymbol{g}^{k}(\boldsymbol{y})\right\|>0
$$

(3)there exists an uncountable subset $S_{0} \subset S$ such that for any $\boldsymbol{x}, \boldsymbol{y} \in S_{0}$,

$$
\liminf _{k \rightarrow \infty}\left\|\boldsymbol{g}^{k}(\boldsymbol{x})-\boldsymbol{g}^{k}(\boldsymbol{y})\right\|=0 .
$$

The anticontrol of chaos for arrhythmia can be realized by choosing appropriate controller parameters based on the above chaotic theory.

\section{Conclusions}

The control and anticontrol of chaos in arrhythmia problem is considered in this paper. The chaotic dynamic model is set up through experimental modeling method. The BP neural network are employ to realize the simulation modeling process, At last, the impulsive differential theory is introduced to complete the control and anticontrol of chaos for cardiac arrhythmias.

\section{Acknowledgements}

This work is supported by the Scientific Research Fund of Liaoning Provincial Education Department under Grant L2012374.

\section{References}

[1] J. W. Dean and M. J. Lab, J. H. Lan., vol. 1, no. 1309, (1989).

[2] P. A. Mohammad, Nonlinear Dynamics, vol. 78, no. 2129, (2014).

[3] V. Vembarasan and P. Balasubramaniam, Nonlinear Dynamics, vol. 78, no. 2031, (2004).

[4] J. G. Lu, Chaos, Solit. \& Fract., vol. 25, no. 403, (2005).

[5] Q. F. Chen, Q. H. Zhong and Y. G. Hong, J. Bifur. Chaos, vol. 17, no. 243, (2007).

[6] J. Li, F. Liu and Z. H. Guan, Neurocomputing, vol. 117, no. 33, (2013).

[7] Y. Q. Yang, G. J. Wang and Y. Yang, J. Mach. Learning \& Cybern., vol. 5, no. 815, (2014).

[8] X. J. Gao, T. W. Huang and Z. Y. Wang, Cogn. Comput., vol. 6, no. 331, (2014). 
[9] T. C. Bai, H. B. Meng and J. H. Yang, Neural Comp. Appl., vol. 25, no. 1699, (2014).

[10] C. P. Li and G. R. Chen, Chaos, Solit. \& Fract., vol. 18, no. 69, (2003).

\section{Author}

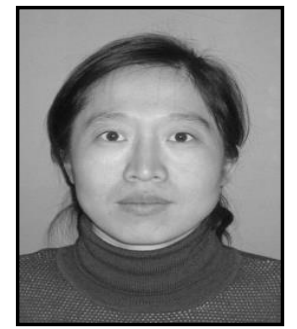

Lihua Miao, she received the Master degree in Physics from Shenyang Normal University, Shenyang, China, in 2006.

She is currently an associate professor with the Department of Computer and Mathematics \& Physics, Shenyang Medical College, Shenyang. Her current research interests include chaotic control in medical field and neural networks theory. 
International Journal of $u-$ and e- Service, Science and Technology Vol.8, No. 6 (2015) 